\title{
A NOTE ON A LIMITING BEHAVIOUR OF THE OCCURRENCE TIMES OF A MIXED POISSON PROCESS
}

\author{
PETER ALBRECHT,* University of Mannheim
}

In a recent publication Pfeifer (1982) shows that for a Pólya-Lundberg birth process the limiting distribution of the sequence $\left\{n / T_{n}\right\}$, where $T_{n}$ denotes the $n$th occurrence time of the process, is a gamma distribution. As the Pólya-Lundberg birth process is a mixed Poisson process with a gamma mixing distribution, Pfeifer's result implies that the limiting distribution of $\left\{n / T_{n}\right\}$ is just the mixing distribution of the process. A stronger result of this type is valid for every mixed Poisson process, which can be seen as follows. Given a random variable $\Delta$ whose distribution is the mixing distribution of the process, we notice, following Grandell $((1976)$, p. 12), that the process $\bar{N}(t)=N(t \Delta)$, where $N(t)$ is a Poisson process with unit intensity, is a version of the desired mixed Poisson process. Hence

$$
P\left(\lim _{t \rightarrow \infty} \frac{\bar{N}(t)}{t}=\Delta \mid \Delta=\lambda\right)=P\left(\lim _{t \rightarrow \infty} \frac{N(\lambda t)}{t}=\lambda\right)=1 .
$$

By integration we obtain $\bar{N}(t) / t \rightarrow \Delta$ a.s. As

and

$$
\frac{\bar{N}(t)}{t} \leqq \frac{n}{T_{n}} \text { for } \quad T_{n} \leqq t<T_{n+1}
$$

$$
\frac{n}{T_{n}} \leqq \frac{n}{n-1} \frac{\bar{N}(t)}{t} \text { for } \quad T_{n-1} \leqq t<T_{n},
$$

we have $n / T_{n} \rightarrow \Delta$ a.s. and also $T_{n} / n \rightarrow 1 / \Delta$ a.s.

I thank a referee for pointing out an easier proof of an even more general result.

\section{References}

Grandell, J. (1976) Doubly Stochastic Poisson Processes. Lecture Notes in Mathematics 529, Springer-Verlag, Berlin.

PFEIFER, D. (1982) An alternative proof of a limit theorem for the Pólya-Lundberg process. Scand. Actuarial J., 176-178.

Received 31 January 1983; revision received 22 March 1983.

* Postal address: Institut für Versicherungswissenschaft. Universität Mannheim, D-6800 Mannheim, Schloß, West Germany. 